C-A/AP/\#316

July 2008

\title{
Polarization Issues in Run 2008
}

S. Y. Zhang, L. Ahrens, H. Huang, K. Zeno

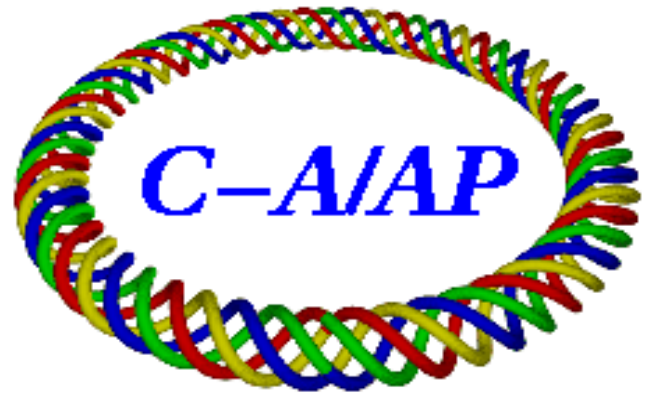

Collider-Accelerator Department

Brookhaven National Laboratory

Upton, NY 11973

Notice: This document has been authorized by employees of Brookhaven Science Associates, LLC under Contract No. DE-AC02-98CH10886 with the U.S. Department of Energy. The United States Government retains a non-exclusive, paidup, irrevocable, world-wide license to publish or reproduce the published form of this document, or allow others to do so, for United States Government purposes. 


\title{
Polarization Issues in Run 2008
}

\author{
S.Y. Zhang, L. Ahrens, H. Huang, and K. Zeno \\ July 17,2008
}

\begin{abstract}
The RHIC proton beam polarization has a strong dependence on intensity in Run 2008, whereas the dependence is almost absent in Run 2006. Meanwhile, the RHIC beam transverse emittance also has a dependence on intensity in Run 2008, but little in Run 2006. Using the emittance measurement at the AGS IPM and the BtA multiwires, the source of this difference between 2006 and 2008 runs is traced to the Booster. It is found that at least the degree of the vertical scraping in the Booster is different in 2006 and 2008. The effect of this scraping for the RHIC beam emittance and polarization is studied.
\end{abstract}

\section{RHIC polarization and emittance depen- dence on intensity}

\subsection{Polarization dependence on intensity}

In the polarized proton run 2008 (pp08), the polarization dependence on intensity is observed. This is different from the pattern observed in the polarized proton run 2006 (pp06).

In Fig.1, the polarization versus bunch intensity in RHIC is shown for pp06 and pp08. The average bunch intensity is taken at the end of the RHIC beam injection, which is about $95 \%$ of the AGS extraction intensity. The polarization is taken at the RHIC early store. There are total 131 fills of pp06, and 46 fills of pp08.

The circle in pp06 represents fills after the one-week shutdown, which was taken place around April 20, 2006. After the shutdown, at least the 


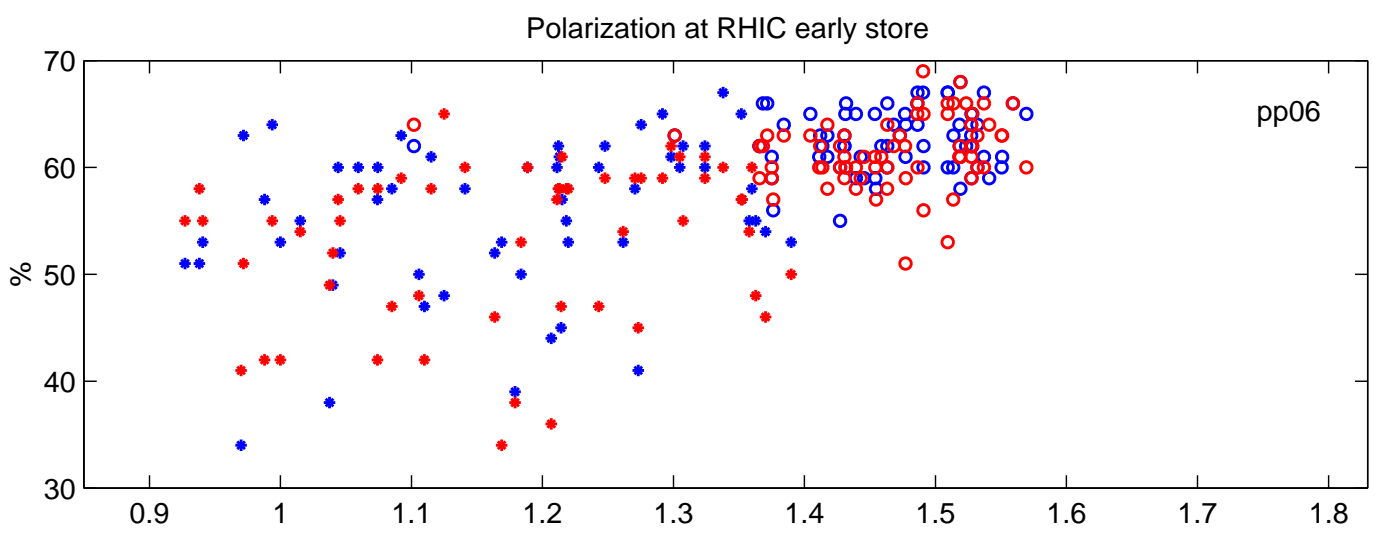

Blue: Blue ring, Red: Yellow ring

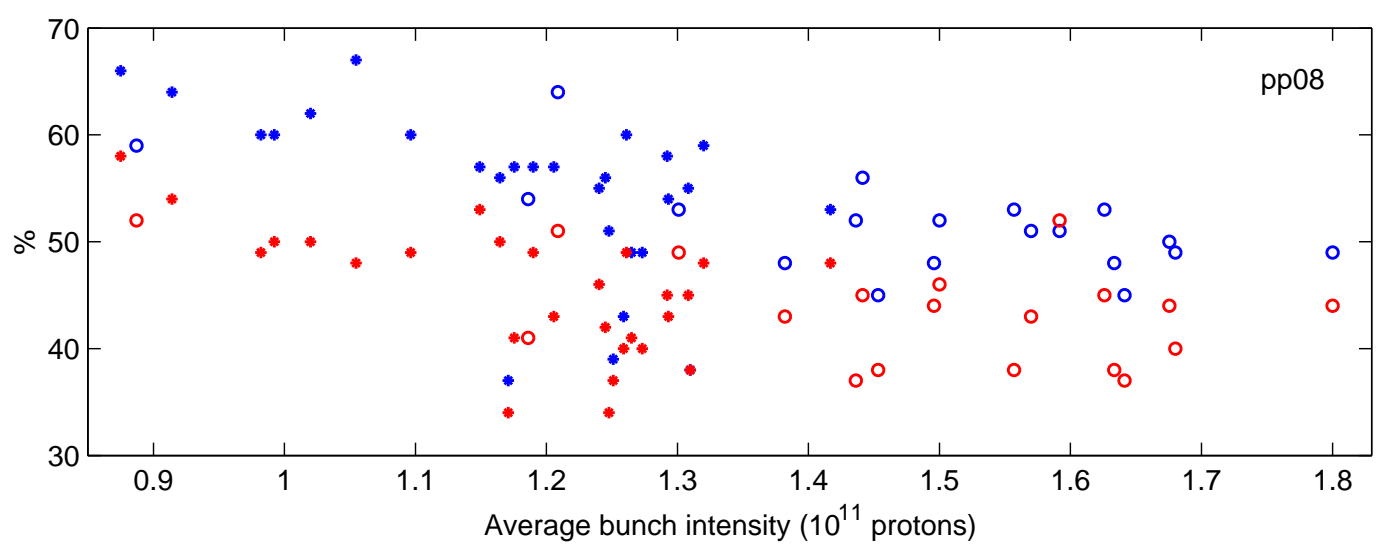

Figure 1: RHIC beam polarization from online data versus average bunch intensity in pp06 and pp08. Circle in pp06 represents fills after the one-week shutdown, and in pp08 represents fills after the AGS is changed from User 4 to User 2. There is no polarization dependence on intensity in pp06, but the dependence is strong in pp08. 
AGS extraction lattice was changed. The circle in pp08 represents fills after the AGS is changed from User 4 to User 2, i.e., the AGS is changed from injection-on-the-fly to injection with a dwell field. The AGS User 2 is the same as the one used for later pp06.

The polarization of Blue and Yellow beams in RHIC is very similar in pp06. In pp08, the Yellow polarization is consistently lower than Blue in RHIC, which is likely to be a problem in RHIC, and will not be discussed in this note.

The polarization in RHIC has little dependence on intensity in pp06, but the dependence is strong in pp08. In specific, for bunch intensities from $10^{11}$ to $1.8 \times 10^{11}$ protons, the best polarization of blue beam in RHIC is reduced from $65 \%$ to $50 \%$ in pp08.

\subsection{Beam emittance dependence on intensity}

In Fig.2, the normalized beam emittance, derived from the ZDC coincident rate, versus bunch intensity in RHIC is shown for pp06 and pp08.

The beam emittance in pp06 is evaluated by the PHENIX ZDC coincident rate at 1.5 hours after the acceleration started. For pp08, it is evaluated after the beam just getting into collision. Since the interest here is in the emittance dependence on intensity, the calibration of the emittance will not be further elaborated.

The beam emittance in RHIC has no dependence on intensity in pp06, but the dependence is strong in pp08. Specifically, in pp08, for bunch intensities from $10^{11}$ to $1.8 \times 10^{11}$ protons, the emittance is increased from $14 \pi \mu \mathrm{m}$ to $20 \pi \mu m$.

The source of the emittance dependence on intensity in pp08 needs to be investigated.

\section{Emittance dependence on intensity is traced to the Booster}

\subsection{AGS beam emittance}

For many RHIC fills, the AGS IPM measurement around the time of the RHIC injection is available in the e-log. In Fig.3, the RHIC beam emittance is shown together with the AGS IPM measurement for pp06 and pp08. For 

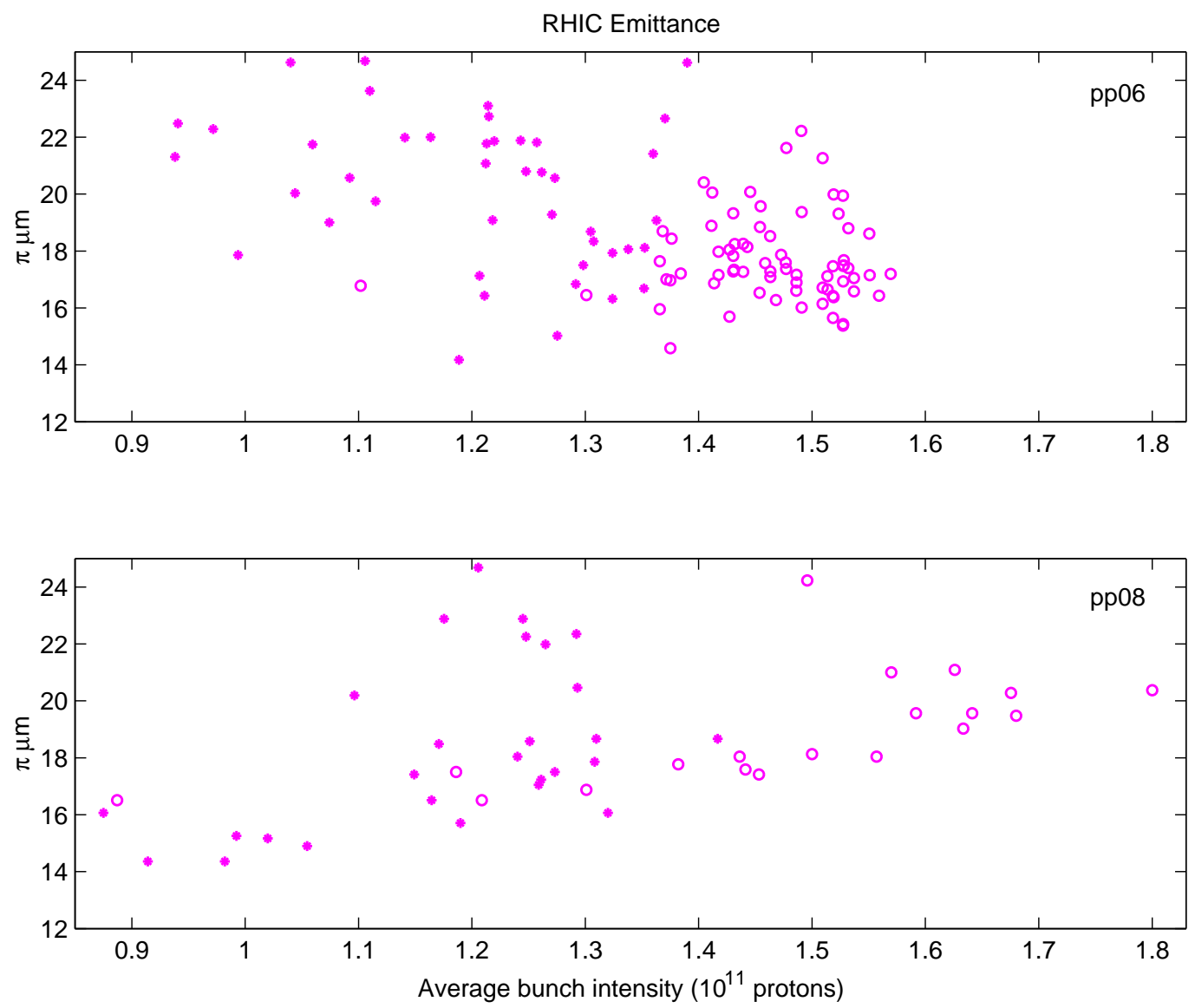

Figure 2: Normalized RHIC beam emittance versus average bunch intensity in pp06 and pp08. Circle in pp06 represents fills after the one-week shutdown, and in pp08 represents fills after the AGS is changed from User 4 to User 2. There is no emittance dependence on intensity in pp06, but the dependence is strong in pp08. 
the AGS IPM measurement, the emittance is taken at $810 \mathrm{~ms}$ from AGS T0 with the RF off, as usual.

The typical horizontal / vertical emittance measured by the AGS IPM after the one-week shutdown in pp06 changed from $13 \pi \mu m / 12 \pi \mu m$, to $11 \pi \mu m / 15 \pi \mu m$. After the shutdown, the bunch intensity in RHIC was increased from $1.3 \times 10^{11}$ to approximately $1.6 \times 10^{11}$ protons, but the AGS IPM emittance kept constant. In Fig.3, only the pp06 AGS IPM measurement after the one-week shutdown is shown.

In pp08 run, after the AGS User 2 is applied, the pattern of the AGS emittance measurement is very similar to later pp06. This might be due to the fact that the AGS lattice is identical to that in later pp06 run. In Fig.3, only pp08 AGS IPM measurement with AGS User 2 is shown.

In Fig.4, the AGS emittance averaged for horizontal and vertical is used to compare with the RHIC emittance.

From Figs.3 and 4, one may find that the emittance dependence on intensity at the AGS is similar to RHIC, i.e., this dependence is absent in pp06, but is strong in pp08.

\subsection{BtA beam emittance}

An emittance scan with the Booster beam intensity at the Booster to AGS (BtA) transfer line shows that source of the AGS emittance dependence on intensity might not be in the AGS, but in the Booster.

The measurement took place in the later pp08 run, with the Booster late intensity of $0.95 \times 10^{11}, 1.8 \times 10^{11}, 2.6 \times 10^{11}$, and $3.4 \times 10^{11}$ protons. Normalized by the highest intensity, the vertical beam size is $0.73,0.80$, $0.90,1.0$, and horizontal is $0.82,0.87,0.93,1.0$. At the time, the Booster late intensity of $3.4 \times 10^{11}$ protons approximately yields $1.8 \times 10^{11}$ protons in RHIC, and the emittance at this intensity is about $15 \pi \mu \mathrm{m}$. Taking these assumptions, in Fig.5, the BtA beam emittance measurement is shown together with the RHIC and AGS beam emittance. One may conclude that the emittance dependence on intensity is similar for that at RHIC, AGS, and BtA as well.

The emittance dependence on intensity in pp08 is, therefore, traced to the Booster. 


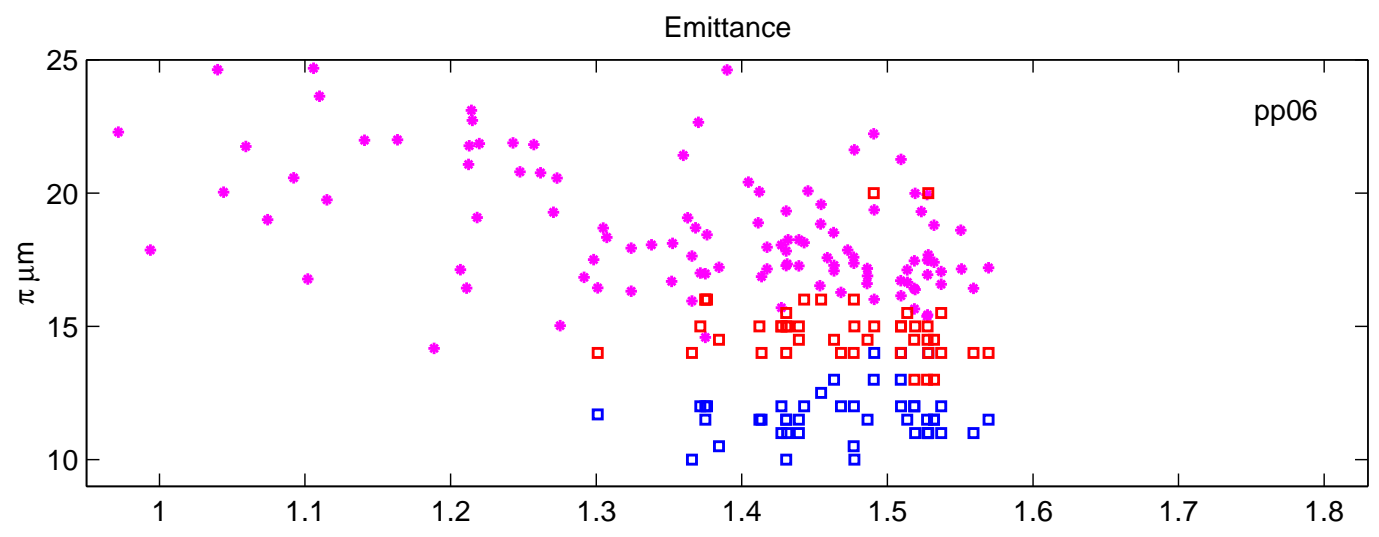

Magenta: RHIC. Red: AGS V, Blue: AGS H

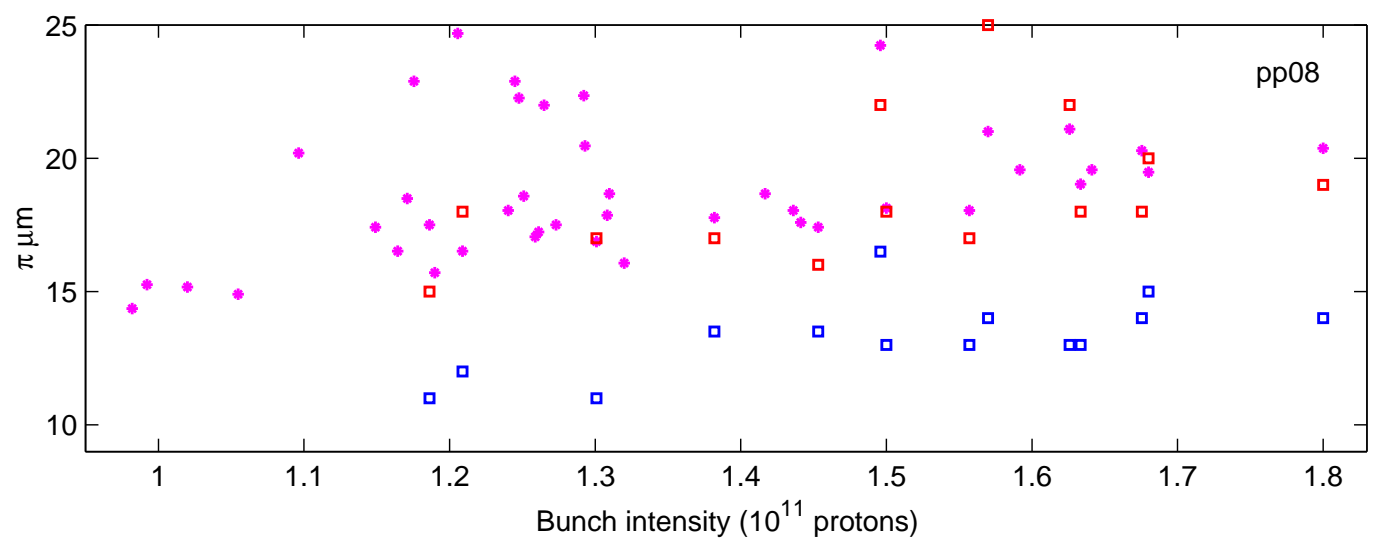

Figure 3: RHIC beam emittance derived from the ZDC coincident rate is compared with the horizontal and vertical emittance measured by AGS IPM for pp06 and pp08. Magenta is for RHIC emittance. Blue and red for AGS horizontal and vertical emittance, respectively. 


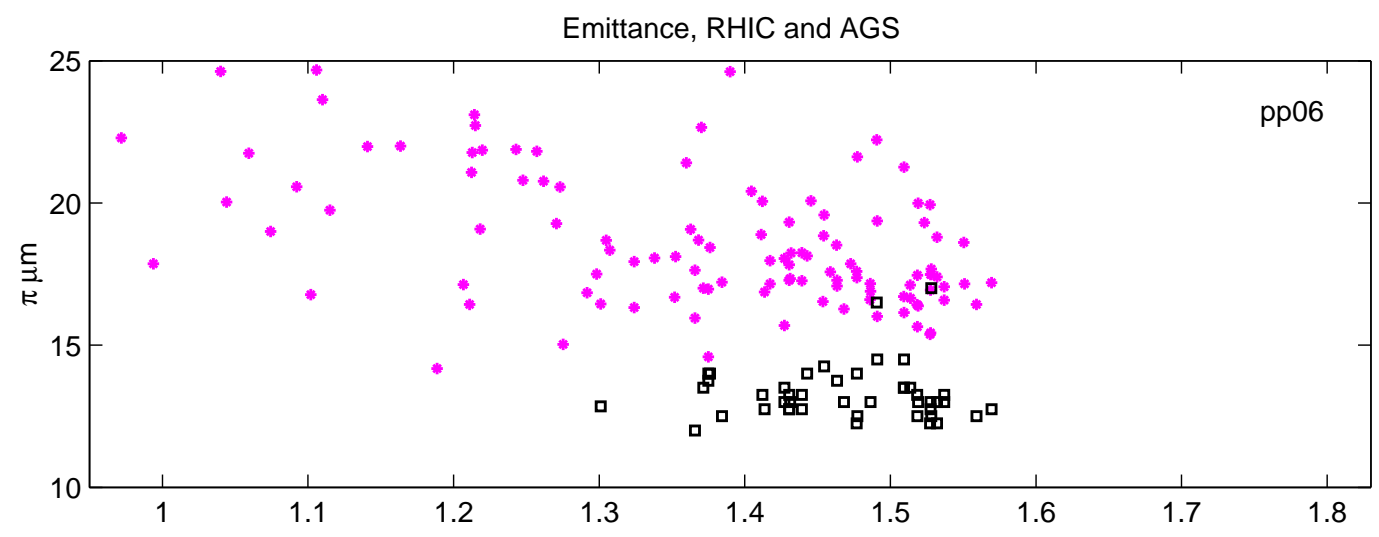

Magenta: RHIC, Black: AGS

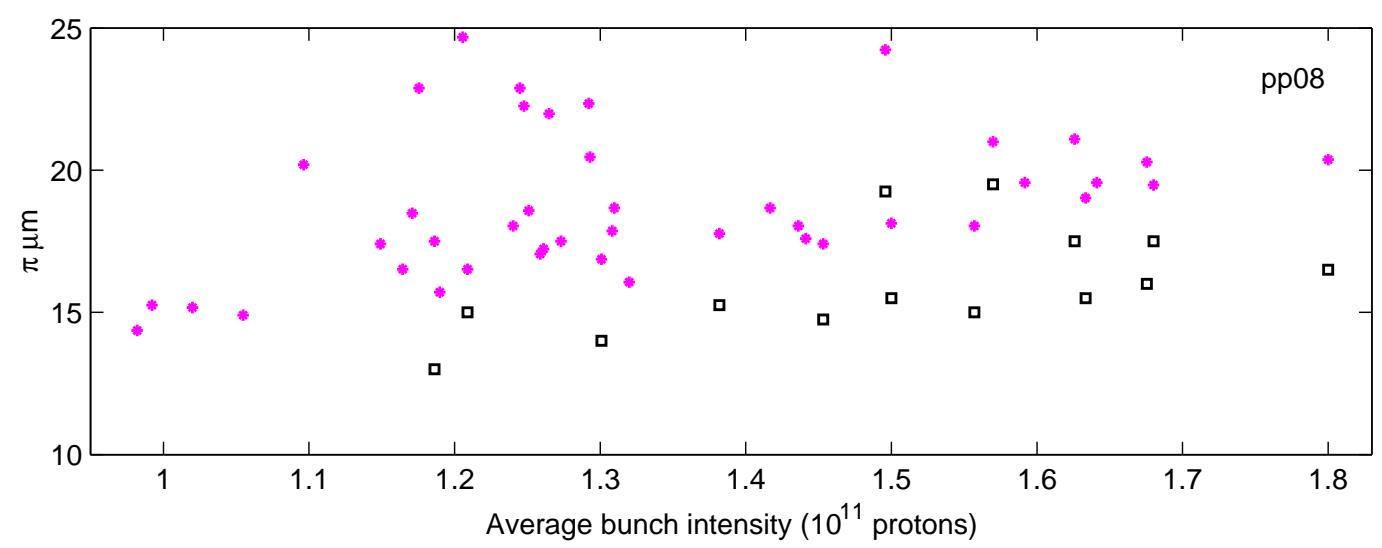

Figure 4: RHIC beam emittance is compared with the average emittance (horizontal and vertical) measured at AGS for pp06 and pp08. Magenta is for RHIC emittance. Black is the average of AGS horizontal and vertical emittance. 
RHIC, AGS and BtA Emittance

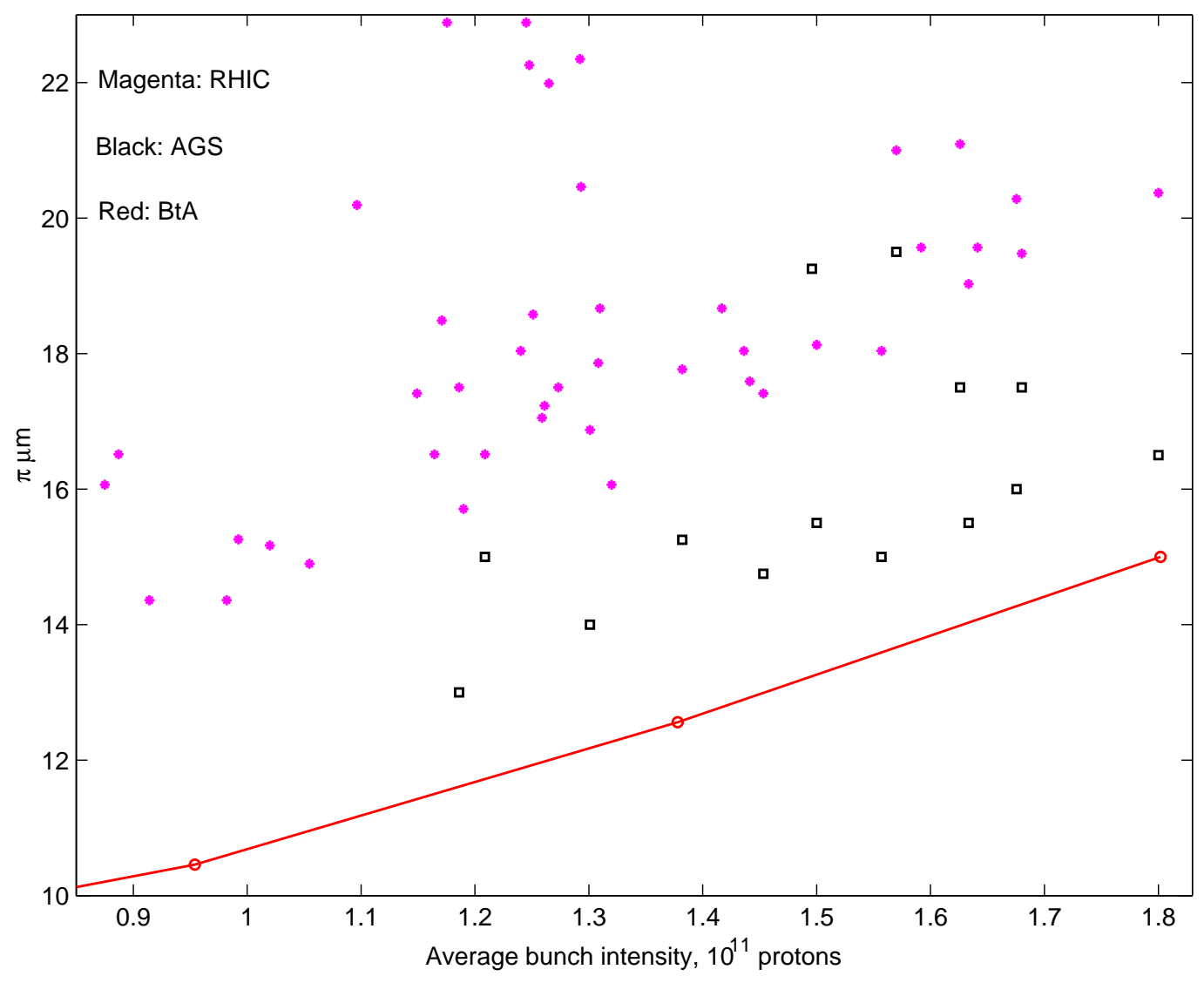

Figure 5: The beam emittance measured at the BtA has similar dependence on intensity with the RHIC and AGS in pp08. 

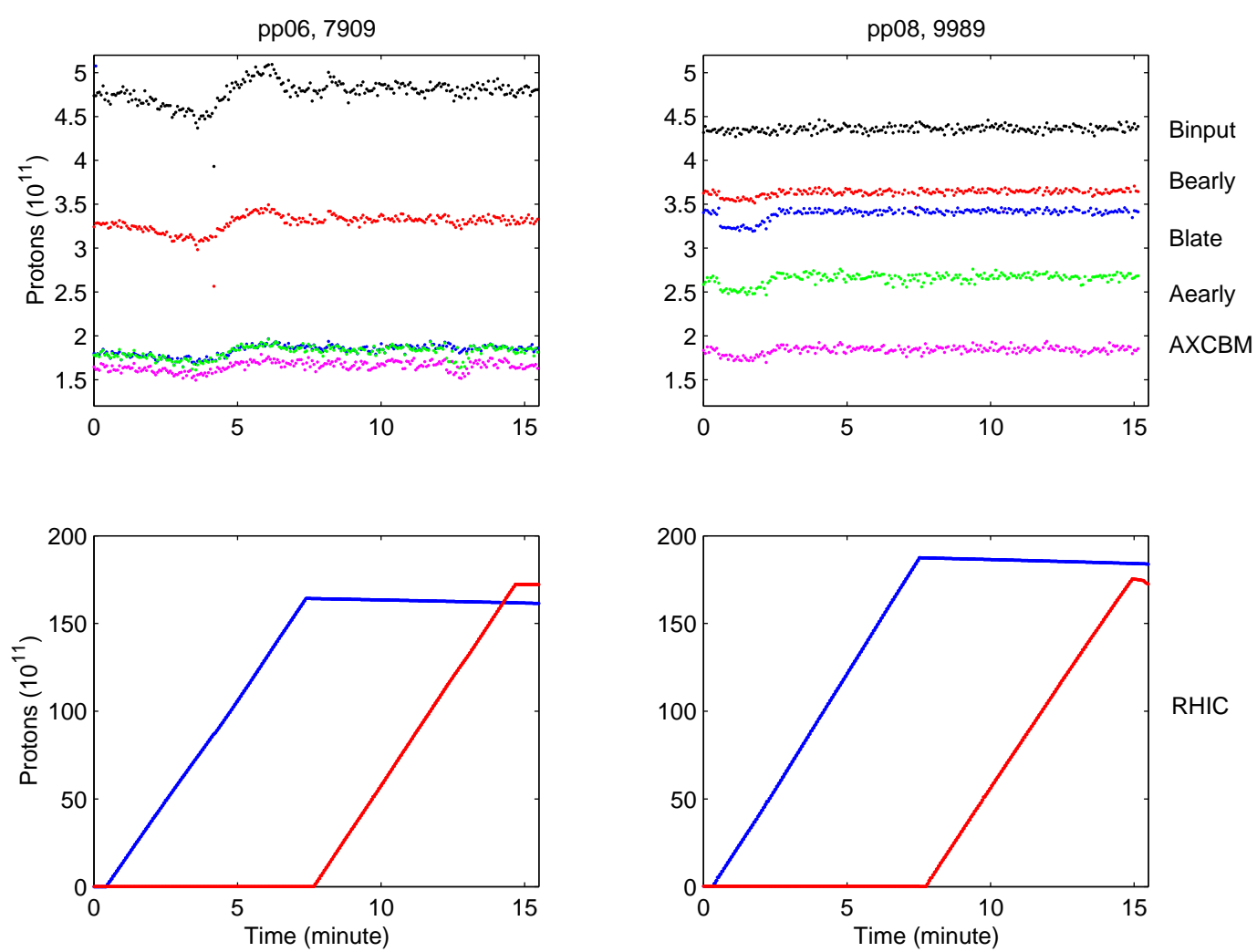

Figure 6: Booster settings are compared for golden fills 7909 in pp06 and 9989 in pp08. Binput is Booster input, Bearly is Booster early, Blate is Booster late, Aearly is AGS early, and AXCBM is at the AGS extraction.

\subsection{Booster settings}

The settings in the Booster can be compared for the golden fills 7909 in pp06 and 9989 in pp08. A golden fill is the one with the highest luminosity in entire run. Also to be mentioned that the fill 7909 is after the one-week shutdown in pp06, and the fill 9989 is after changed to AGS User 2 in pp08. The total beam intensity at the Booster input (Binput), Booster early (Bearly), Booster late (Blate), AGS early (Aearly), and at the AGS extraction (AXCBM) are shown in Fig.6.

In the Booster settings, the injection chopper width and the vertical scraping are perhaps the two most important features. The larger chopper width implies more turns of the Booster injection, which usually yields higher inten- 
sity at Binput. The heavier Booster vertical scraping means larger difference from the Binput to the Blate.

Given the similar Binput, for 7909 in pp06 and 9989 in pp08, the ratio of Blate / Binput is very different, which means the Booster vertical scraping settings are different.

The transmission from Blate to AXCBM, which includes the Booster extraction, BtA transfer, the AGS injection and acceleration, is different in pp06 and pp08. In fact, the transmission from Blate to AXCBM in pp06 is much better than pp08. The better transmission is explained by the smaller beam size with more vertical scraping in pp06, which helped to reduce the beam loss during the course.

In Fig.7, the AXCBM is plotted against Blate for fills 7847 to 7912, two weeks in later pp06, and for fills 9954 to 10002, two weeks in later pp08. For pp06, the AXCBM of $2 \times 10^{11}$ protons is corresponding to approximately $2.2 \times 10^{11}$ protons at Blate, indicating about $90 \%$ of transmission. In pp08, the same AXCBM needs about $3 \times 10^{11}$ protons in Blate, indicating some $66 \%$ of transmission.

It is therefore of interest to take a close look at the effects of the Booster settings.

\section{$3 \quad$ Effects of Booster vertical scraping}

In Fig.8, the RHIC beam polarization, the RHIC beam emittance, and the ratio of AXCBM/Blate are shown for almost the entire pp06 run. As discussed previously, in general, more Booster vertical scraping leads to better transmission from Booster late to AGS XCBM. Therefore, the larger the $\mathrm{AXCBM} /$ Blate ratio, the more the Booster vertical scraping.

For the early pp06, i.e., for fills 7568 to 7720 , the ratio of AXCBM/Blate was increased from $<0.6$ to $>0.8$, which implies the increasing Booster vertical scraping. At the same period of time, the polarization in RHIC was improved, from $<50 \%$ to around $60 \%$, and the beam emittance in RHIC was reduced by some $10 \pi \mu m$, which is a significant amount.

A closer look at the early pp06 run offers more insight into the effect of the Booster settings. This is shown in Fig.9. For about a dozen fills centered at the fill 7650, the Booster scraping is heavy and the ratio of Blate/Binput is reduced to $<0.4$. Meanwhile, the transmission from Blate to AXCBM is improved to $>0.8$. With fairly high Booster input intensity, the RHIC 


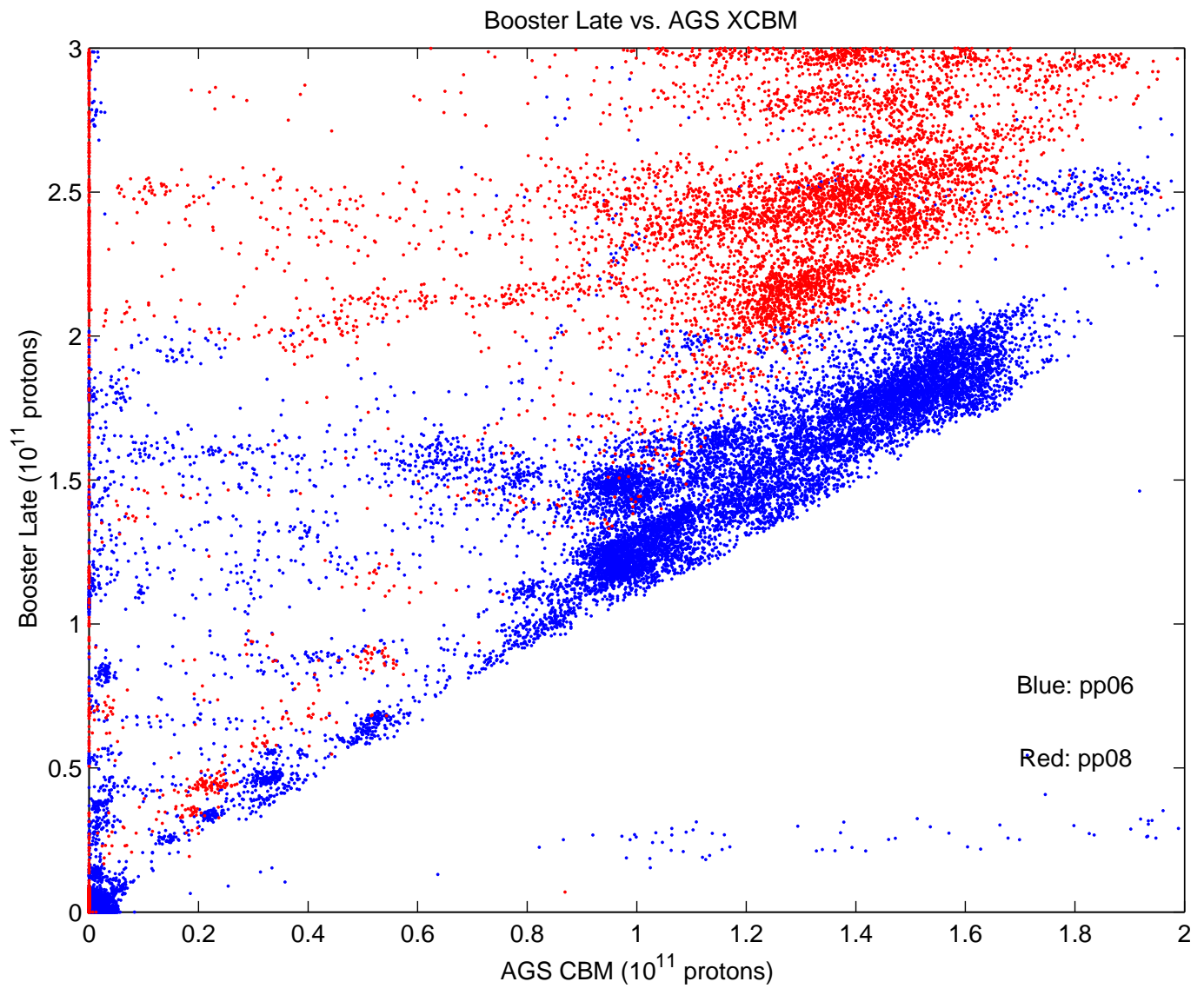

Figure 7: AGS XCBM versus Booster late for two weeks each in later pp06 (blue) and pp08 (red). 

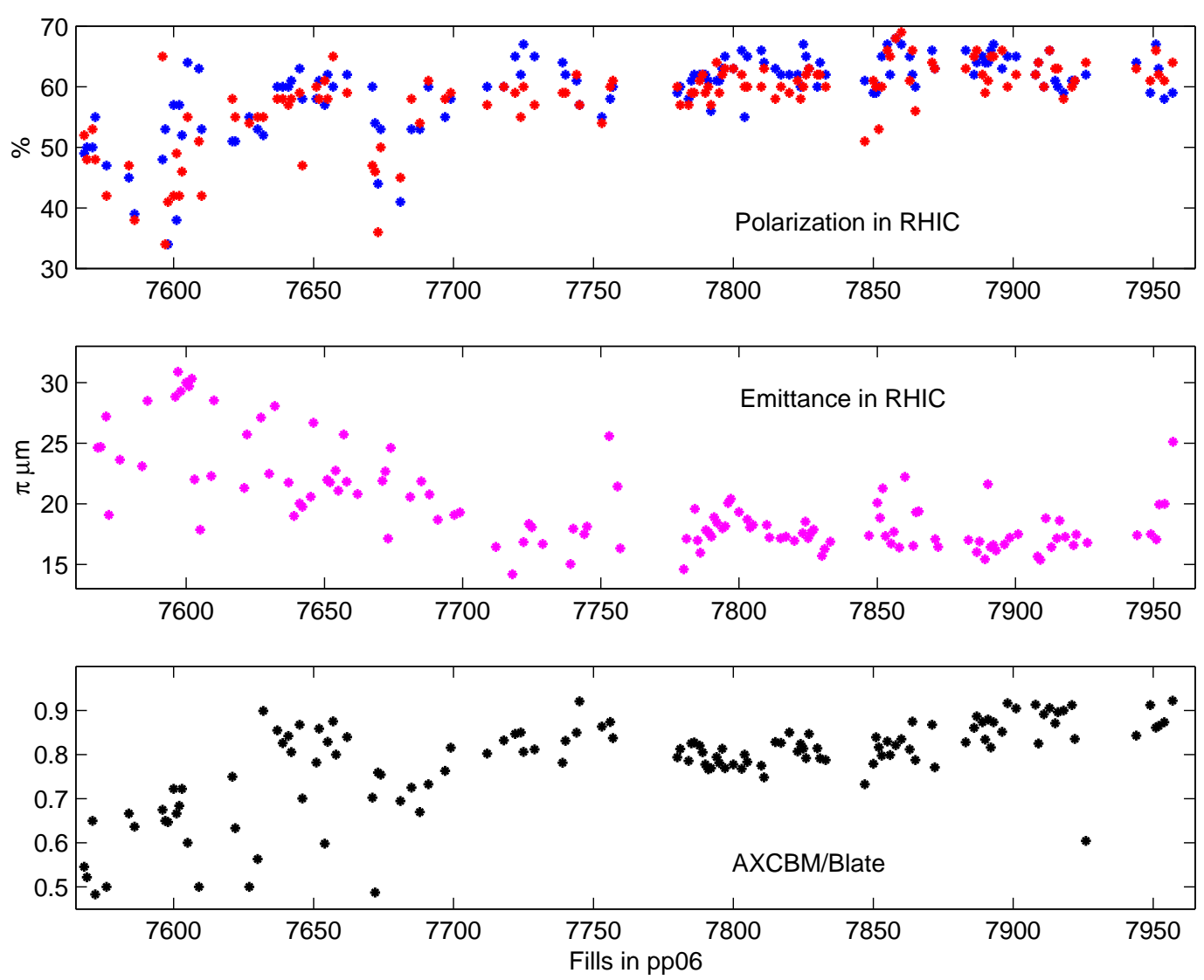

Figure 8: Effect of Booster vertical scraping, represented by AXCBM/Blate, on the RHIC polarization and emittance for pp06. 

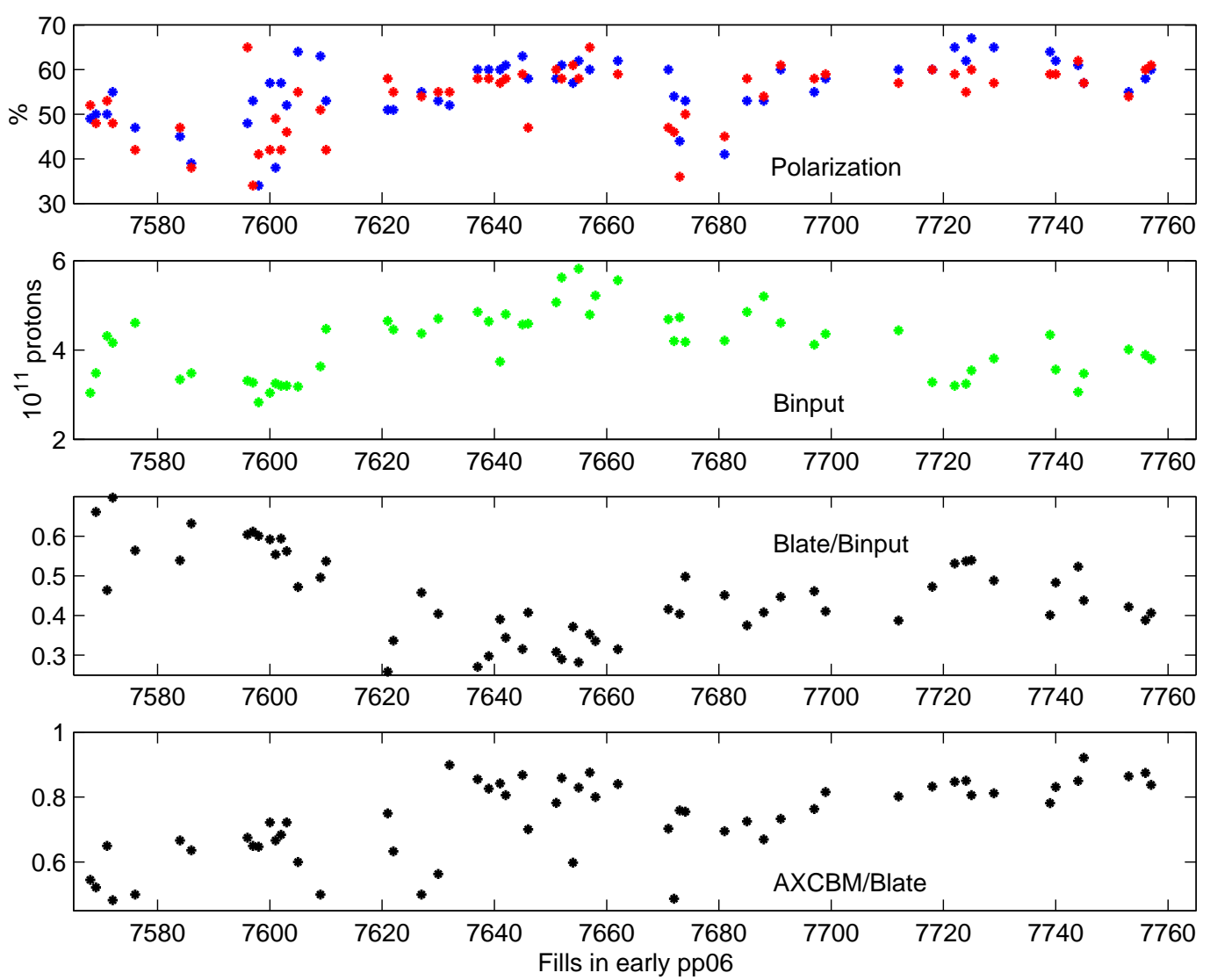

Figure 9: Effect of the Booster vertical scraping, represented by both Blate/Binput and AXCBM/Blate, early pp06.

polarization is peaked around $60 \%$. This shows the effect of the Booster vertical scraping on the polarization.

For 5 fills centered at 7722, the Booster vertical scraping is rather modest and the ratio of Blate/Binput is around 0.5. The polarization is high, around $60 \%$, and the reason of this might be the low Booster input intensity, about $3.5 \times 10^{11}$ protons. The low intensity usually leads to better polarization, but it has less interest in the need to push up the RHIC luminosity.

In Fig.10, it is further shown that more Booster vertical scraping may improve the polarization, despite the increased Booster input intensity.

For fills 7760 to 7850 , the Booster input is increased from $4 \times 10^{11}$ to $5.5 \times 10^{11}$ protons, mainly by setting for larger chopper width, i.e., with 

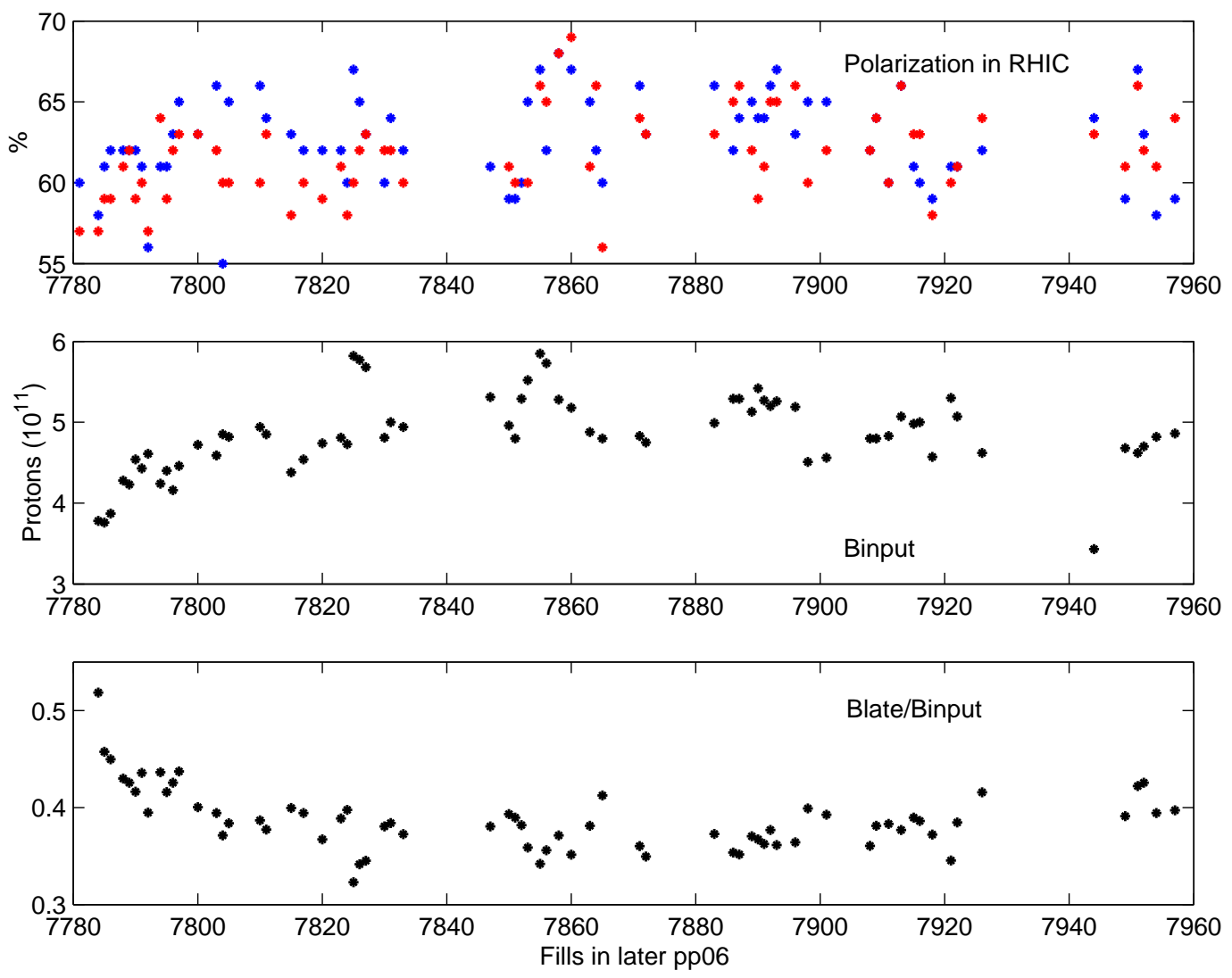

Figure 10: Effect of the Booster vertical scraping, represented by Blate/Binput, later pp06. 

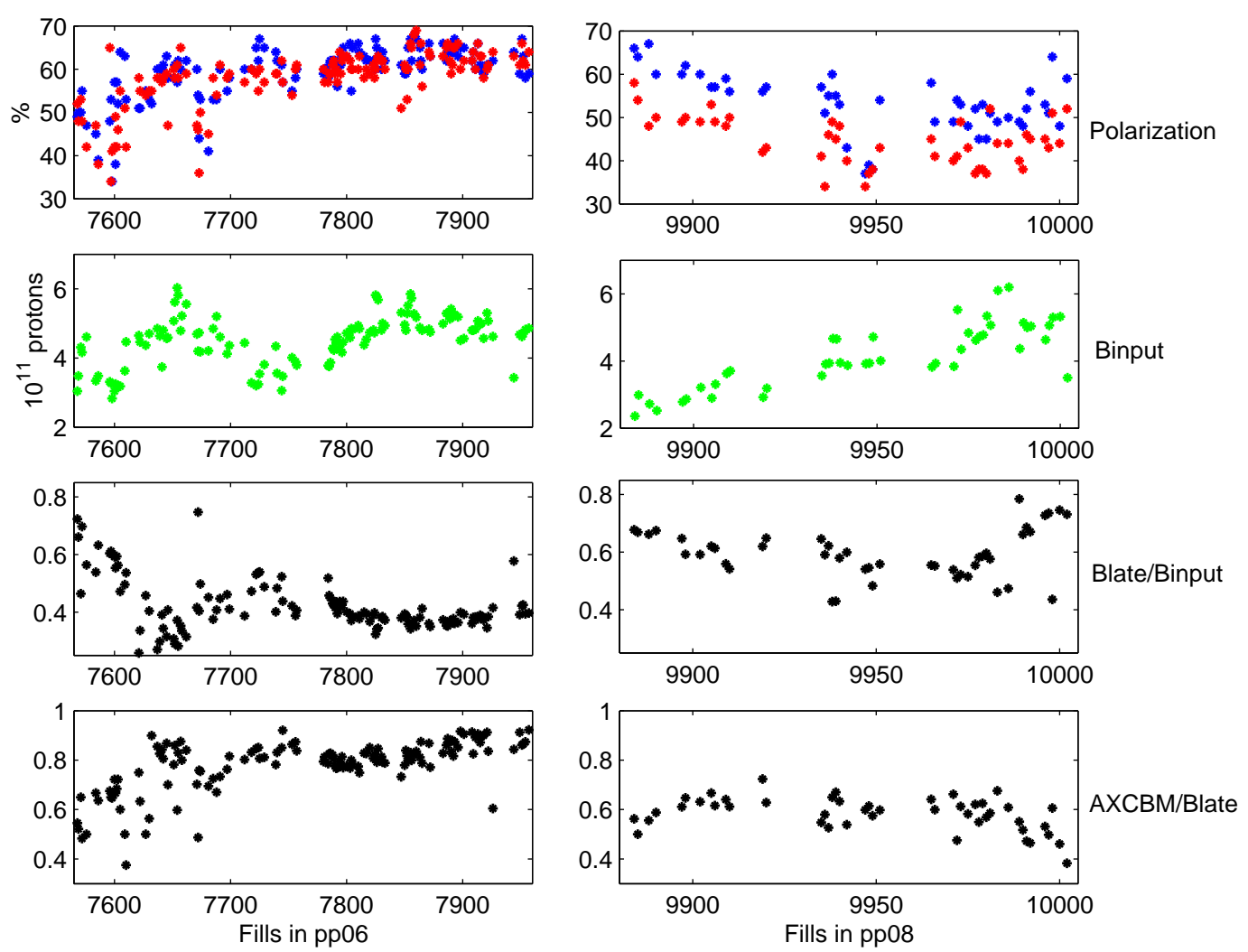

Figure 11: History of Booster vertical scraping and the effect on the polarization of pp06 and pp08.

more turns of beam injections. The Booster vertical scraping was increased during the same time, from about 0.5 to $<0.4$ in the ratio of Blate/Binput. The RHIC polarization is gradually improved from $<60 \%$ to about $65 \%$, and meanwhile the AXCBM is increased by about $10 \%$. It is shown, at least in this case, the benefit of the Booster vertical scraping on polarization is more than offsets the negative effect of the higher Booster input intensity.

Finally, in Fig.11, the polarization, the Booster input, the ratio of Booster late/Booster input, and the ratio of AGS XCBM/Booster late are compared for pp06 and pp08. Taking the middle parts in the two runs, the typical Blate/Binput is 0.4 in pp06, but it is 0.6 in pp08. Consequently, the typical AXCBM/Blate is 0.8 in pp06, but it is 0.6 in pp08. 


\section{Discussion}

The polarization and emittance in RHIC have a dependence on intensity in the polarized proton run 2008, whereas this dependence is almost absent in the 2006 run. Using the AGS IPM and BtA multipwire measurements, the emittance dependence on intensity is traced to the Booster.

There is a significant difference between the Booster vertical scraping settings in the 2006 and 2008 runs. During the pp06 run, the Booster vertical scraping was gradually increased, which resulted in better polarization and smaller emittance in RHIC. In pp08, the Booster vertical scraping was never applied to the comparable degree as that in pp06. Once switched to AGS User 2 in pp08, only 11 days left to the end of run. Also, the smaller dependence of the beam emittance on the intensity with the AGS User 2, compared with User 4, allowed beam intensity push with some $70 \%$ increase of the luminosity, which was the main focus during that period of time. This effort might have affected the tuning of the Booster vertical scraping.

In Fig.12, the Booster vertical scraping, represented by the ratio of Blate/Binput, versus the transmission from Blate to AXCBM in pp06 and pp08 are shown. The general agreement between the two runs can be observed, but a cluster of pp08 fills with relatively heavier Booster scraping is worth more attentions. The ratio Blate/Binput of these fills is between 0.4 to 0.5, and the transmission of AXCBM/Blate is between 0.6 to 0.7. With similar degrees of the Booster scraping, the best transmission in pp06 can be $>0.8$. These fills in pp08 are having Booster input intensities from $4.6 \times 10^{11}$ to $6.2 \times 10^{11}$ protons, and taken place before and after the AGS changed to User 2. These fills are: 9938, 9939, 9949, 9983, 9986, 9998.

It seems that the Booster vertical scraping may improve the polarization through the reduction of the emittance. It is not clear, however, where and how much the de-polarization takes place during the course: Booster acceleration, AGS injection, and AGS acceleration. Some study is desired.

With the luminosity goal in-sight, one needs to push for the high intensity at the AGS. Although with more Booster scraping the later beam transmission is improved, there might be a limit of the Booster scraping.

The Booster horizontal scraping may affect the protons with large momentum deviation, and hence the beam longitudinal profile. A systematic study of the horizontal scraping may help for a better understanding.

At present, the estimate of the beam emittance at the Booster extraction is between 10 to $15 \pi \mu m$, whereas the LINAC beam injected into the Booster 


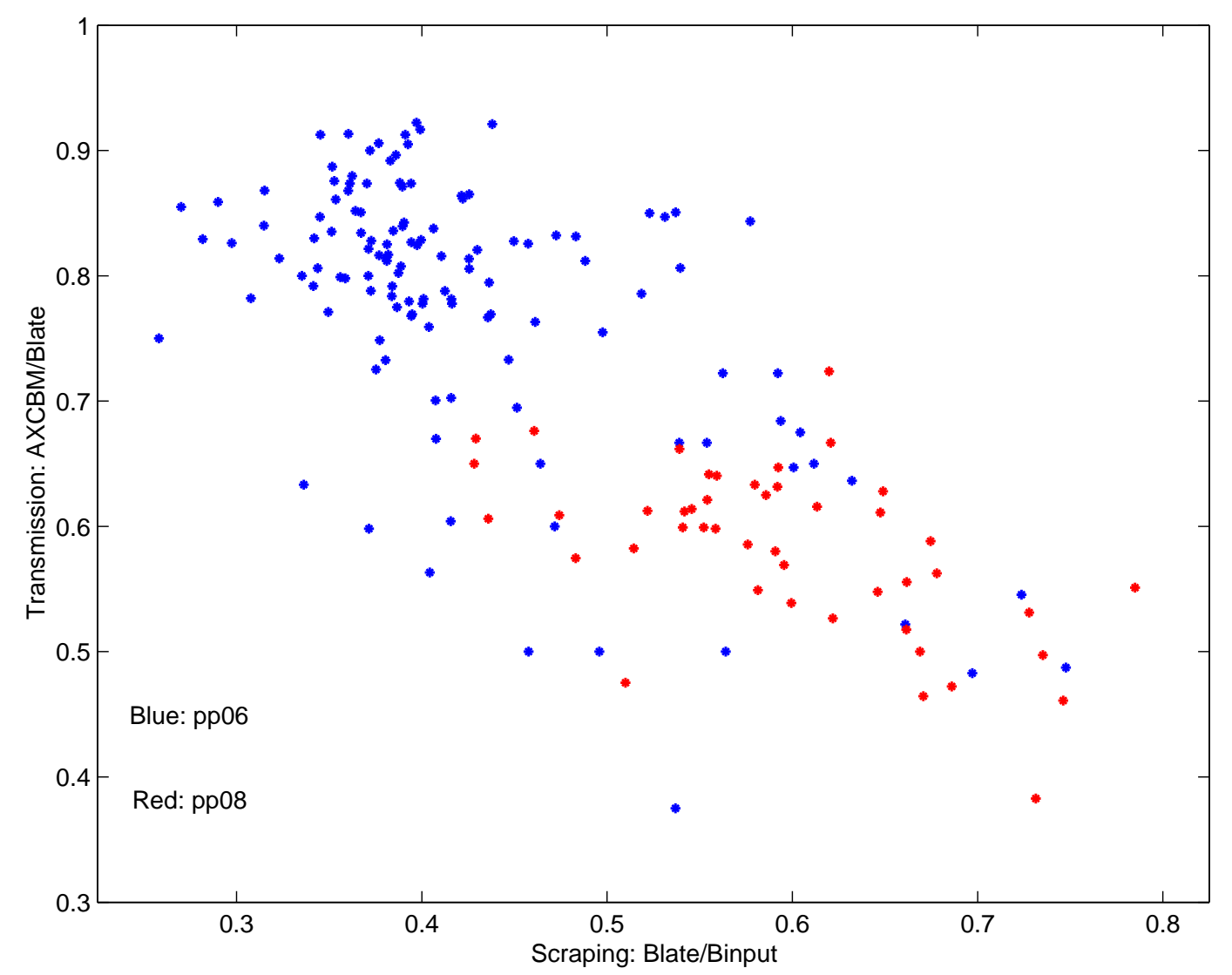

Figure 12: Compare Booster vertical scraping (Blate/Binput) with the transmission from Blate to AXCBM (AXCBM/Blate) for pp06 and pp08. 
with some 5 to $10 \pi \mu m$. The reduction of the LINAC beam emittance is certainly of interest. 